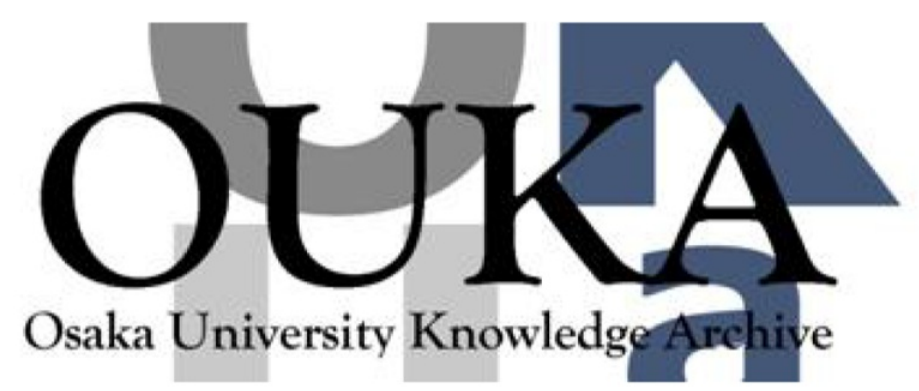

\begin{tabular}{|c|l|}
\hline Title & $\begin{array}{l}\text { Experimental studies of the advanced fast } \\
\text { ignitor scheme }\end{array}$ \\
\hline Author(s) & $\begin{array}{l}\text { Norreys, P. A.; Allott, R.; Clarke, R. J. et } \\
\text { al. }\end{array}$ \\
\hline Citation & Physics of Plasmas. 7(9) p. 3721-p. 3726 \\
\hline Issue Date & $2000-09$ \\
\hline oaire:version & VoR \\
\hline URL & https://hdl. handle.net/11094/3260 \\
\hline rights & \\
\hline Note & \\
\hline
\end{tabular}

Osaka University Knowledge Archive : OUKA

https://ir. Library. osaka-u. ac. jp/

Osaka University 


\title{
Experimental studies of the advanced fast ignitor scheme
}

\author{
P. A. Norreys, R. Allott, R. J. Clarke, J. Collier, D. Neely, and S. J. Rose \\ Rutherford Appleton Laboratory, Chilton, Didcot, Oxon OX11 0QX, United Kingdom
}

M. Zepf, M. Santala, A. R. Bell, K. Krushelnick, and A. E. Dangor

Blackett Laboratory, Imperial College of Science, Technology and Medicine, Prince Consort Road, London SW7 2BZ, United Kingdom

\author{
N. C. Woolsey and R. G. Evans \\ Department of Physics, University of York, Heslington, York YO1 5DD, United Kingdom \\ H. Habara, T. Norimatsu, and R. Kodama \\ Institute of Laser Engineering, Osaka University, 2-6 Yamada-oka, Suita, Osaka 565, Japan
}

(Received 25 April 2000; accepted 10 May 2000)

\begin{abstract}
Guided compression offers an attractive route to explore some of the physics issues of hot electron heating and transport in the fast ignition route to inertial confinement fusion, whilst avoiding the difficulties associated with establishing the stability of the channel formation pulse. X-ray images are presented that show that the guided foil remains hydrodynamically stable during the acceleration phase, which is confirmed by two-dimensional simulations. An integrated conical compression/fast electron heating experiment is presented that confirms that this approach deserves detailed study. (C) 2000 American Institute of Physics. [S1070-664X(00)02809-3]
\end{abstract}

\section{INTRODUCTION}

The possibility of producing high energy gain using inertial confinement fusion is the subject of intense study around the world. Most notable is the work toward achieving gain using indirectly driven targets on the National Ignition Facility, currently being constructed at the Lawrence Livermore National Laboratory, USA (see the paper by Lindl ${ }^{1}$ for a detailed review and all references therein). The concept of fast ignition has been proposed as a means of achieving the same result as the conventional scheme, but using much less drive energy. ${ }^{2}$ Fast ignition involves the compression of the deuterium-tritium (DT) fuel in the normal way, but rather than rely on accurate shock timing to ignite the fuel, ignition is generated by an ultraintense, multi-PetaWatt laser beam. After compression by the long-pulse beams, a channel is formed in the plasma atmosphere, requiring laser intensities of $10^{17}-10^{19} \mathrm{~W} \mathrm{~cm}^{-2}$ for the duration of several hundred picoseconds. During this "hole-boring" phase the critical surface is pushed toward the dense core. At the stagnation point of the implosion an "ignitor" pulse $\left(\sim 10^{21} \mathrm{~W} \mathrm{~cm}^{-2}\right.$, $\sim 10$ ps with an energy of tens of kiloJoules) is driven into the channel formed by the "hole boring" and deposits energy at the critical surface with high conversion to high energy electrons. These electrons propagate into the DT core and are stopped, leading to strong local heating and the ignition of a fusion burn wave. ${ }^{3-5}$

There are plainly many aspects of the fast ignition scheme that need further study, but one particular problem appears to be the "hole boring" phase, which risks instabilities in both the ablation hydrodynamics and laser propagation. The ability to produce a channel that remains empty and stable for long enough that the ignitor beam can pass up it has been seriously questioned. This problem is being vigorously addressed experimentally. ${ }^{6,7}$
To avoid this difficulty altogether, an alternative fast ignition scheme has been suggested that removes the holeboring phase of the process by requiring that the initial implosion does not take place in spherical geometry. Two possibilities have been considered (although a variety of other geometries suggest themselves once the restriction of a spherical implosion is lifted). These are illustrated in Fig. 1. The first (a) requires the implosion of a spherical shell that is almost complete, but involves a gold cone which keeps the channel clear for the short-pulse beam to reach the imploded plasma at stagnation. ${ }^{8}$ The second method (b) is much more speculative and requires that the implosion take place down a cone and the ignitor beam interacts with the imploded plasma at the base of the cone. ${ }^{9}$ In this case the presence of the fast ignitor relaxes the constraints on $\rho$ and $\rho R$ of the compressed fuel and opens up the design space, but with the danger of mixing high $Z$ material into the burn region. In each case these advanced fast ignition concepts have effectively traded a plasma physics problem (the hole-boring problem) for a hydrodynamics problem (an implosion in the presence of a cone).

Experiments performed at the Central Laser Facility this year have investigated one of these advanced fast ignition concepts. These experiments, described in Secs. IV and V, have tested aspects of the physics underlying the scheme using an implosion driven down a cone. We chose the geometry in Fig. 1(b) rather than Fig. 1(a) because it is impossible to drive the target in (a) with the long pulse energy available with the VULCAN Nd glass laser (a few kilojoules). ${ }^{10}$ The attraction of the target in Fig. 1(b) for this experiment was that it reduced the long-pulse laser energy required by a factor of about 10 , allowing the experiment to be undertaken on VULCAN whilst providing us with experimental information on the hydrodynamic behavior of shells 
(a)

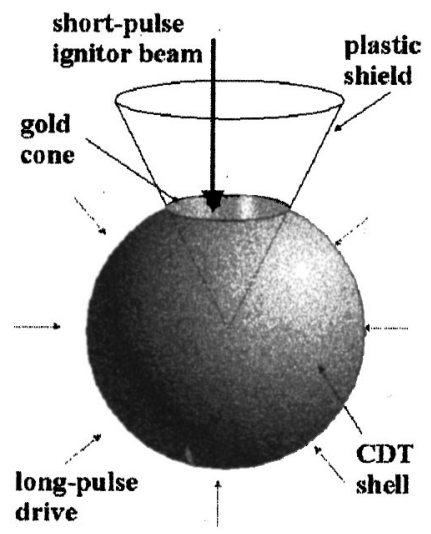

(b)

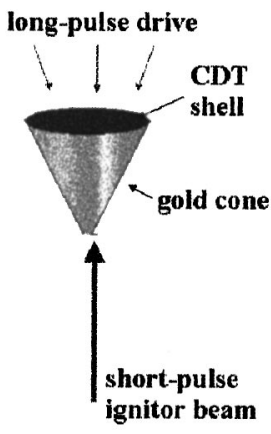

FIG. 1. Schematic illustration of the advanced fast ignitor concept showing (a) the implosion of a CDT shell with a guide cone for the fast ignitor beam, (b) a conical compression guided by high $Z$ walls, allowing complete access for the fast ignitor beam to the plasma at the peak compression of the guided foil.

driven down cones. This is essential for the evaluation of both schemes in Fig. 1. Indeed, experiments have been performed recently that have demonstrated that electron beam direction can be precisely pointed with the careful control of the plasma density scale length. ${ }^{11-16}$ The advanced fast ignition scheme, which is the subject of this paper, provides a much shorter scale length for the interaction of the ignitor pulse with the plasma than in the original fast ignition

scheme. It thus offers the opportunity for much more precise control of the energy deposition process, in addition to these other benefits.

\section{MODELING}

The preliminary guided compression experiments that we have conducted on the VULCAN laser have been modeled using the Eulerian code POLLUX. ${ }^{17}$ POLLUX is a twodimensional hydrodynamic code that uses a corrected Thomas-Fermi equation of state, although no radiation transport is included in the calculations. Simulations were performed with $240 \times 133$ cells on a PC class machine. The target and illumination geometry was varied to assess implosion quality with a Gaussian shaped laser pulse. A further set of simulations was performed to determine the effect of shaped laser pulses on the guided compression of flat foil targets.

The entrance hole of the cone was set at $200 \mu \mathrm{m}$ diameter, and the depth of the cone from the entrance hole to the apex was $250 \mu \mathrm{m}$. This was matched to the available laser energy of the VULCAN Nd glass laser. The foil was set at $12 \mu \mathrm{m}$ thickness, and the drive beam was a $2 \mathrm{~ns} 527 \mathrm{~nm}$ pulse at an intensity of $5 \times 10^{14} \mathrm{~W} \mathrm{~cm}^{-2}$. The guided compression of an initially flat foil target is illustrated in Figs. 2(a)-2(c). Flat foils gave a poorer performance than spherical foils in compressed density, $\sim 3 \mathrm{~g} \mathrm{~cm}^{-3}$, due to two problems. The first is that associated with assembling all the foil elements at the apex simultaneously (each foil element along (a)

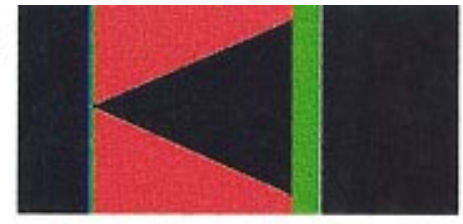

(b)

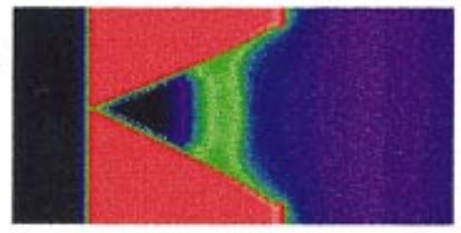

(c)

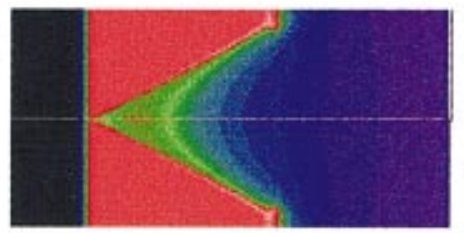

(d)

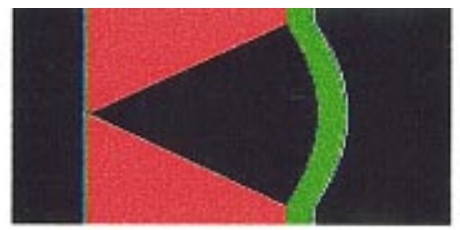

(e)

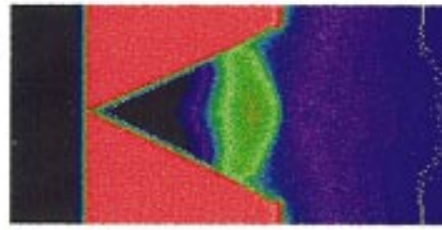

(f)

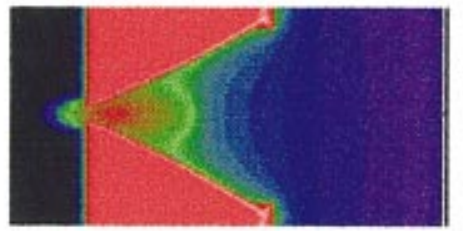

(g)

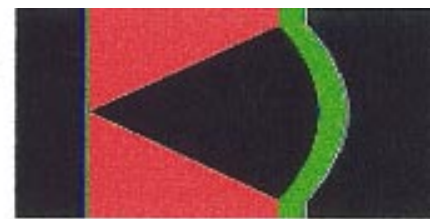

(h)

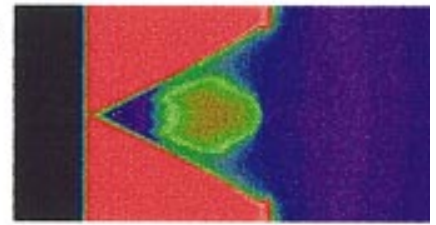

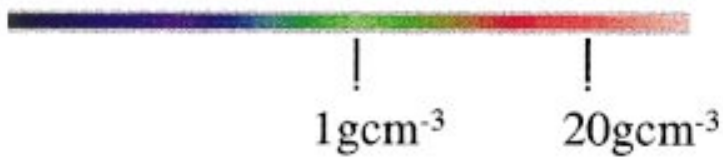

FIG. 2. (Color) POLLUX simulations of the temporal evolution of the guided conical compression showing (a) an initially flat foil target, (b) a later snapshot of the guided compression showing roll up of plasma on the walls of the cone, and (c) the guided plasma at its highest density. (d) A spherical shaped foil target with a radius of curvature that is matched to the radius of the conical section, (e) a later snapshot of this foil showing reduced roll up of the guided foil on the edges of the cone and (f) the compressed plasma at its highest density for this spherical foil target. (g) A spherical foil target with a radius of curvature that is smaller than the radius of the conical section, (h) the position of the guided plasma at its highest density has moved into the cone. 
the entrance hole has a different distance to the apex). The second is that a reflected shock propagates from the boundary between the Au guide material and the plastic $(\mathrm{CH})$ foil due to the nonparallel propagation of the different materials. The reflected shock increases the pressure in the $\mathrm{CH}$ material near the boundary and consequently the guided material rolls up at the edges [Fig. 2(b)]. The roll up potentially mixes the material from the high $Z$ guide and the compressed foil, which is most undesirable.

This mixing problem can be greatly reduced with the use of spherically shaped foils. Figures $2(\mathrm{~d})-2(\mathrm{~h})$ show the guided compression of spherical shaped cap targets whose curvature is (i) matched to the radius of the cone [Figs. 2(d)2(f)] and (ii) is greater than the radius of the cone so that the center of the spherical cap is inside the cone [Figs. 2(g) and 2(h)]. The highest peak densities of $10 \mathrm{~g} \mathrm{~cm}^{-3}$ were achieved in the simulations for the matched spherically shaped foils. The compression of over-curved foils demonstrates that the peak compression of the guided plasma can be adjusted to be inside the guide cone, but at the cost of reduced densities.

The shaping of initially flat foils with controlled laser illumination from random phase plates was also studied. In this case, the intensity on the foil at the edge of the cone was higher than at the center by ratios of up to $2: 1$. This made little difference to the shape of the imploding foil due to the large standoff distance between the ablation front and the critical density surface that results in significant thermal smoothing of the imposed intensity profile.

In the second set of simulations, a $1 \mathrm{~ns}$ foot pulse at $1 / 10$ of the intensity of the drive pulse preceded the main 2 ns drive beam. In this case, it was found that densities of 20 $\mathrm{g} \mathrm{cm}^{-3}$ were obtained for flat foils, but only when the main 2 ns laser pulse was at an intensity $5 \times 10^{14} \mathrm{~W} \mathrm{~cm}^{-2}$. When the intensity was reduced to $1 \times 10^{14} \mathrm{~W} \mathrm{~cm}^{-2}$ densities $\sim 3$ $\mathrm{g} \mathrm{cm}^{-3}$ were again obtained.

It can therefore be concluded that spherically shaped foils and shaped laser pulses give the best performance in terms of compressed densities and $\rho R$. These simulations reinforce the conclusions of earlier experiments performed in the late 1970s at Los Alamos ${ }^{18}$ and Moscow's Lebedev Institute. ${ }^{19-21}$ These early investigations gave encouraging preliminary results, but these experiments have not been repeated.

\section{LASER CONFIGURATION}

The first question to address in guided compression is the effect of the high $Z$ walls on the stability of the foil during the compression phase. To study this interesting stability problem, an experiment was conducted on the Nd:glass laser VULCAN. The laser was configured such that the output of the broad-bandwidth $(16 \mathrm{~nm})$ Tsunami Ti-S oscillator was stretched to $300 \mathrm{ps}$ with additional amplification before being split between the two rod amplifier arms. This eliminated all timing jitter between the different beam lines, and provided broad bandwidth for spectral smoothing in the long pulse drive beams. In the long pulse arm, a beam splitter was used to stack two pulses to provide a 600 ps full width at half maximum duration pulse. The ratio between the two pulses

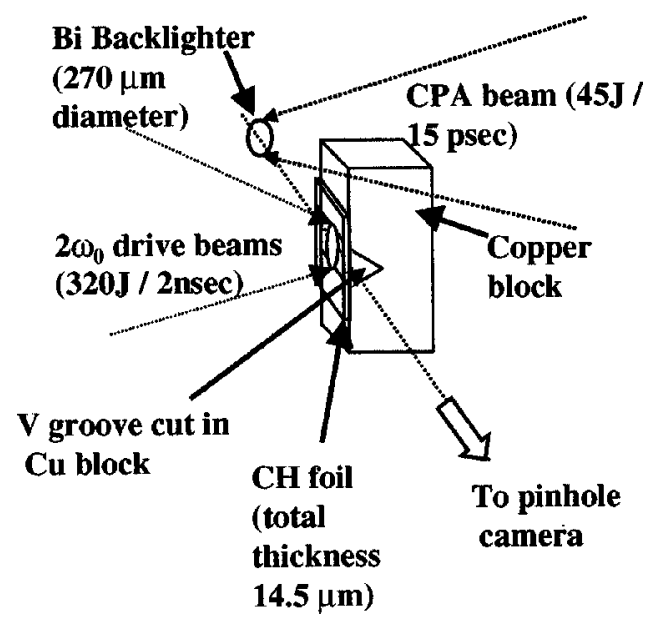

FIG. 3. Schematic of the "v-groove" backlight experiment.

in the beam splitter was adjusted to provide a flattop output pulse; i.e., the effect of gain saturation in the laser amplifier was taken into account on the final pulse shape. This pulse was then amplified in the rod chain and then split between the six $108 \mathrm{~mm}$ disk amplifiers. All long pulse beams were frequency doubled to $527 \mathrm{~nm}$. Two beams were stacked to provide a foot pulse of $16 \mathrm{~J}$ in $1 \mathrm{~ns}$ resulting in an intensity of $5 \times 10^{13} \mathrm{~W} \mathrm{~cm}^{-2}$ to target. The remaining four beams were displaced in time to provide a flattop main drive pulse to target of $320 \mathrm{~J}$ in $2 \mathrm{~ns}$ resulting in an intensity of 5 $\times 10^{14} \mathrm{~W} \mathrm{~cm}^{-2}$ on target. Shaped random phase plates, together with $f / 10$ lenses generated a focal spot diameter of $200 \mu \mathrm{m}$. The intensity distribution in the focal plane was doughnut shaped and was higher on the edges than in the center by a factor of 1.2:1.0. The doughnut intensity profile was used in an attempt to shape the flat foil during the implosion (although our simulations have revealed that this was not particularly successful due to thermal smoothing). In the short pulse arm of the VULCAN laser, the beam was amplified to the $60 \mathrm{~J}$ level (45 J to target) and compressed using large area diffraction gratings to $15 \mathrm{ps}$. The beam was focused onto target using an $f / 3$ off-axis parabola and was either used to provide a $\mathrm{x}$-ray backlighter for the radiographic studies (the first "v-groove" experiment) or heat the compressed plasma (in the second "conical" experiment).

\section{V-GROOVE EXPERIMENT}

Figure 3 shows the first "v-groove" experimental layout. The short pulse beam was focused onto a circular aluminum disk (13 $\mu \mathrm{m}$ thickness, $270 \mu \mathrm{m}$ diameter), which was coated with $0.5 \mu \mathrm{m}$ of bismuth. The beam was incident onto target at $30^{\circ}$ from the target normal with a peak intensity of $5 \times 10^{15} \mathrm{~W} \mathrm{~cm}^{-2}$. The Bi backlighting target was placed 700 $\mu \mathrm{m}$ from the edge of a 2-mm-thick copper block which had a $v$ groove cut into the side. The depth of the groove was 250 $\mu \mathrm{m}$ and the entrance size was $200 \mu \mathrm{m}$. On this $\mathrm{Cu}$ block was mounted a foil consisting of $12.5 \mu \mathrm{m}$-thick polyethylene with $2.0 \mu \mathrm{m}$ of chlorinated paralyene coated on the inner edge. The $2 \omega$ drive beams were focused $500 \mu \mathrm{m}$ from the edge of the $\mathrm{Cu}$ block closest to the backlighter target. The 


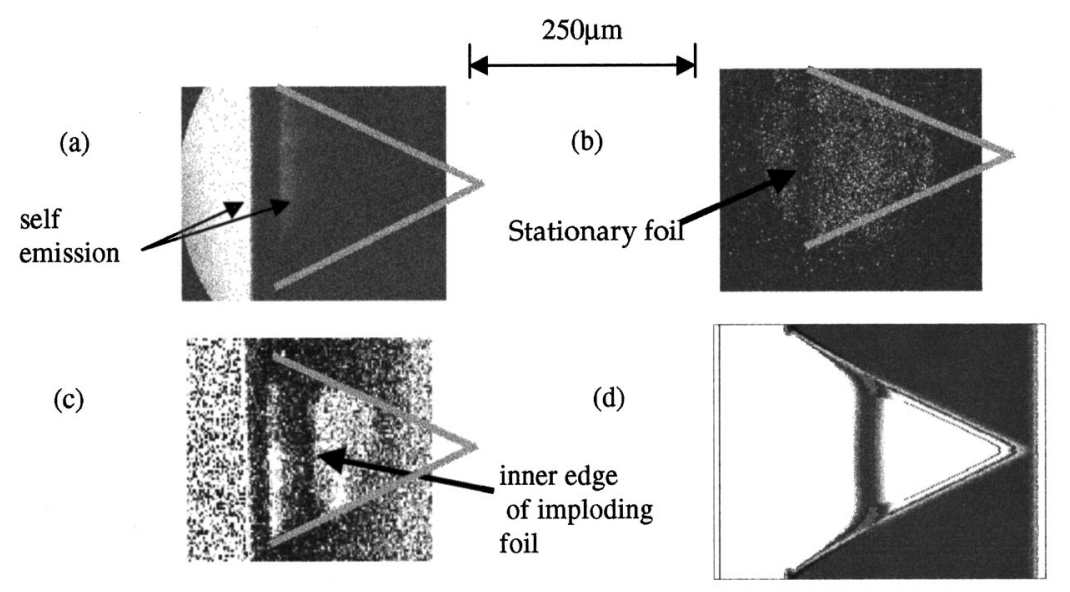

FIG. 4. Pinhole camera images showing (a) selfemission from the foil when only the $2 \omega_{0}$ drive beams were fired, (b) the stationary foil when only the backlighter was fired, (c) the foil being driven into the $\mathrm{v}$ groove taken at $1.0 \mathrm{~ns}$ from the leading edge of the main drive pulse. (d) A 2-D POLLUX simulation taken at this time which shows the roll up of the foil on the edge of the $\mathrm{v}$ groove, in good agreement with the observations.

$5-\mu \mathrm{m}$-diam pinhole was position either at 7 or $17 \mathrm{~mm}$ from the Bi target. The 16 bit Intaspec $\mathrm{x}$-ray charged couple device (which was filtered by $125 \mu \mathrm{m} \mathrm{Be}$ to all eliminate $\mathrm{Cu}$ $L$-shell emission) was positioned $21 \mathrm{~cm}$ from the $\mathrm{Cu}$ target, giving a magnification of $\times 30$ for Figs. 4 (a) and 4(b) and $\times 12$ for Fig. 4(c) below.

Figure 4(a) shows an image recorded when only the $2 \omega$ nanosecond drive beams irradiated the target. The remaining unirradiated stationary foil, which is still in the line of sight, can be seen in this image. Also, some self-emission on either side of the irradiated region from the front and back of the foil can be observed, which is probably associated with the early stages of the acceleration phase. The important point to note is that no self-emission from either the $\mathrm{Cu}$ walls or from the stagnating plasma can be observed. Figure 4(b) shows an image when only the Bi backlighter foil was irradiated. Again, the stationary foil can be seen, together with the area illuminated by the Bi-coated backlighter foil and the $\mathrm{v}$ groove.

Figure 4(c) shows an image of the guided plasma taken $1.0 \mathrm{~ns}$ from the leading edge of the $2 \mathrm{~ns}, 5 \times 10^{14} \mathrm{~W} \mathrm{~cm}^{-2}$ pulse. The self-emission, which is evident in Fig. 4(a), is still visible. Since the drive laser beam profile has an intensity profile that falls off from the edge of the focal region, some of the foil at the two free ends of the foil is accelerated into the $\mathrm{v}$ groove to lower velocities than the material in the bulk of the foil. This lower velocity component at the free edges has absorbed most of the backlighting radiation between the stationary foil and the inner edge. Nevertheless, a close examination of the inner edge of the imploding foil reveals that the inner edge of the foil has moved into the groove. Moreover, the foil is essentially flat over most of its surface and does not yet exhibit the growth of any hydrodynamic instability. However, at the top and bottom of the image, which corresponds to the edges of the guiding $\mathrm{v}$ groove, the foil has obtained some inner curvature and has rolled up on the edge of the $\mathrm{v}$ groove. The rolled up material on the inner edges is also observed in the two-dimensional (2-D) hydrodynamic simulations of the experiment [see Fig. 4(d)]. However, the drive intensity had to be reduced to $1 \times 10^{14} \mathrm{~W} \mathrm{~cm}^{-2}$ in the simulation to match the observed implosion velocity.

\section{CONICAL COMPRESSION EXPERIMENT}

The good agreement between theory and observation encouraged us to perform a more fully integrated conical compression experiment to determine the efficiency of fast electron heating. In this second experiment, the six $2 \omega$ long pulse beams irradiated a $12.5 \mu \mathrm{m}$ flat polyethylene $(\mathrm{CH})$ foil, which was covered with a $2.0 \mu \mathrm{m}$ of deuterated polystyrene (CD) foil. The difficulty of target manufacture prevented the use of spherical shaped foils in this experiment (although advances in target fabrication technology will allow their deployment in future experiments). A 100- $\mu$ m-diam, 100nm-thick signature layer of $\mathrm{Ti}$ and a 500-nm-thick layer of chlorinated paralyene were deposited between the $\mathrm{CH}$ and $\mathrm{CD}$ foils, with the $\mathrm{CD}$ layer facing the apex of the cone. The $\mathrm{Au}$ cone was manufactured using micromachining techniques similar to those used in the fabrication of holhraum targets. The distance from the entrance hole to the apex was $250 \mu \mathrm{m}$ and the $\mathrm{Au}$ cone material was $20 \mu \mathrm{m}$ thick. A 20 $\mu \mathrm{m}$ hole was machined in the apex of the cone, so that the target could be precisely positioned and the fast electrons transport was through the compressed $\mathrm{CH} / \mathrm{CD}$ material only. A $12.5-\mu \mathrm{m}$-thick, $270-\mu \mathrm{m}$-diam polyethylene foil was placed over the apex of the cone, in order to control the scale length of the short pulse interaction process. The $p$-polarized $45 \mathrm{~J} / 15$ ps pulse was focused onto this foil in a $20 \mu \mathrm{m}$ spot, giving a peak intensity on target of $10^{18} \mathrm{~W} \mathrm{~cm}^{-2}$ and electrons with a temperature of between 200 and $300 \mathrm{keV}$. The cone assembly and the foil covering the apex of a sample target is illustrated in Fig. 5.

The diagnostics for this experiment consisted of both time-integrated and time-resolved crystal spectrometers, a highly sensitive Ag activation counter, and a number of current mode time-of-flight neutron detectors. The spatially integrated $\mathrm{x}$-ray emission from the signature layers in the compressed/heated targets was observed using flat crystal spectrometers with a Si 111 crystal for the $\mathrm{Cl} L \alpha(\lambda=4.185$ $\AA)$ and a Si 220 crystal for $\mathrm{Ti} \mathrm{He} \beta(\lambda=2.221 \AA)$ lines. A flat KAP crystal was coupled to a Kentech x-ray streak camera to observe the spatially integrated, but spectrally and temporally resolved $\mathrm{x}$-ray emission. 


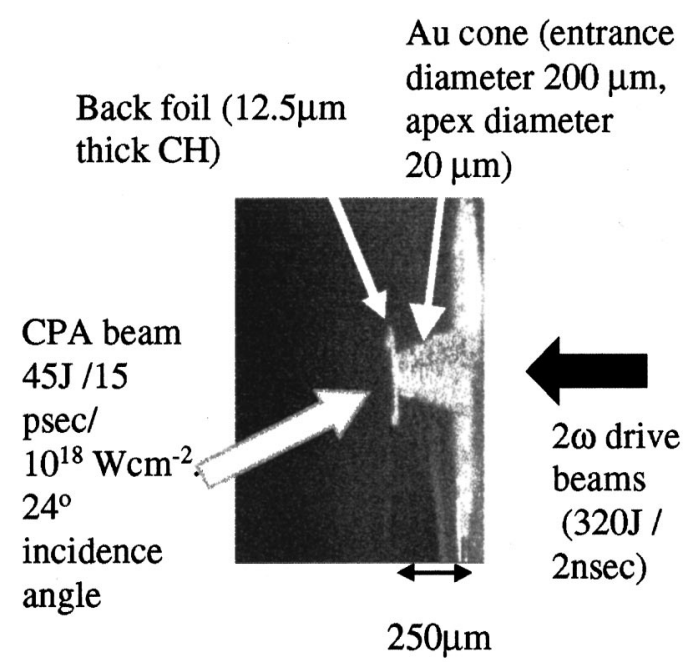

FIG. 5. Experimental arrangement of the conical guided compression experiment.

A highly sensitive Ag activation detector was constructed to measure the $d(d, n)^{3}$ He neutrons from the heated $\mathrm{CD}$ layer. The detector consisted of conical-cylindrical block of high-density polyethylene moderator. Embedded in the moderator was a polished $5 \times 5 \times 10 \mathrm{~cm}^{3}$ perspex block which was surrounded by a 5 -mm-thick NE102 plastic scintillator, the outside of which was covered by $0.5 \mathrm{~mm} \mathrm{Ag}$ foil. The 2.45 MeV neutrons were moderated in the plastics in the detector and the slowed-down neutrons then interacted with the $\mathrm{Ag}$ via the reactions ${ }_{47}^{107} \mathrm{Ag}(n, \gamma){ }_{47}^{108} \mathrm{Ag}$ and ${ }_{47}^{109} \mathrm{Ag}(n, \gamma){ }_{47}^{110} \mathrm{Ag}$. The ${ }_{47}^{108} \mathrm{Ag}$ and ${ }_{47}^{110} \mathrm{Ag}$ then $\beta^{-}$decay to ${ }_{48}^{108} \mathrm{Cd}$ and ${ }_{48}^{110} \mathrm{Cd}$ with a half-lives of 24.6 and 144 seconds, respectively. The $\beta^{-}$particles are stopped in the scintillator and produce a light pulse. The perspex block acts as a waveguide for these photons to the photomultiplier whose electrical signal was then fed into an amplifier/counting setup. The instrument was located such that the perspex block was 10.7 $\mathrm{cm}$ from the target, resulting in a collection angle that was close to $1 \mathrm{sr}$. The instrument was calibrated off-line using a moderated ${ }^{241} \mathrm{Am} / \mathrm{Be}$ neutron source.

The time-resolved $x$-ray spectra showed that there was a significant increase in $\mathrm{x}$-ray emission that peaked at $1.7 \mathrm{~ns}$ after the beginning of the main pulse when only the main drive beams were fired. It suggests that the increase in $\mathrm{x}$-ray emission corresponded to the peak in compressed density of the guided material. Both time-integrated $\mathrm{x}$-ray spectra for this shot did reveal that some $M$-band Au emission was present, as well as some $\mathrm{Cl} L \alpha$ emission from the $\mathrm{Si} 111$ crystal spectrometer, suggesting that kinetic energy of the decelerated foil was converted to thermal energy, as expected. The high intensity heating pulse was then employed and moved to coincide with the stagnation time. The Si 220 crystal spectrometer showed no evidence of time integrated spectra $\mathrm{Ti} \mathrm{He} \beta$ emission when the short pulse beam was fired at $1.7 \mathrm{~ns}$.

Calculations with the atomic physics package FLY ${ }^{22}$ suggest that pressure ionization of the $n=3$ level only becomes significant with densities above $\sim 30 \mathrm{~g} \mathrm{~cm}^{-3}$. Since the densities simulated in POLLUX were an order of magnitude lower than this limit, this effect is not expected to be important in this experiment. The calculations did show, however, that $\mathrm{Ti}$ $\mathrm{He} \beta$ emission should have been observed with an electron temperature of $1.0 \mathrm{keV}$ for densities in the range 1-20 $\mathrm{g} \mathrm{cm}^{-3}$. Thus, the absence of $\mathrm{Ti} \mathrm{He} \beta$ line emission in the experiment sets an upper bound to the heated electron temperature of $\sim 700 \mathrm{eV}$.

Neutrons were observed when the short pulse beam was fired at $1.7 \mathrm{~ns}$. Figure 6(a) shows the $\beta^{-}$decay counts taken in the Ag activation counter for this shot. Also plotted are three other separate shots which were taken when the heating beam was moved $\pm 100 \mathrm{ps}$ from this point in time. These shots show that if any neutrons were generated at these other times, they were below the threshold of the detector (5 $\left.\times 10^{3}\right)$. They tend to rule out $(\gamma, n)$ and $(p, n)$ neutron generation processes in the target or the surrounding equipment, because then the neutrons would have been generated at all times. When the background signal is subtracted from the decay signal, as in Fig. 6(b), the observed $\beta^{-}$decay curve is consistent with a $25 \mathrm{~s}$ half life, and a yield of $2 \times 10^{4}$ neutrons [the signal to noise is too small to unambiguously count the ${ }_{47}^{109} \mathrm{Ag}(n, \gamma){ }_{47}^{110} \mathrm{Ag}$ reaction]. The data strongly suggest that the neutrons are generated as a result of the thermonuclear reaction $d(d, n)^{3} \mathrm{He}$ from the heated $\mathrm{CD}$ layer.

We can make an estimate of the plasma temperature needed to generate the observed neutron yield. Assume the fast electrons uniformly heat the plasma. The energy obtained from an inertially confined DT plasma is equal to the (a)

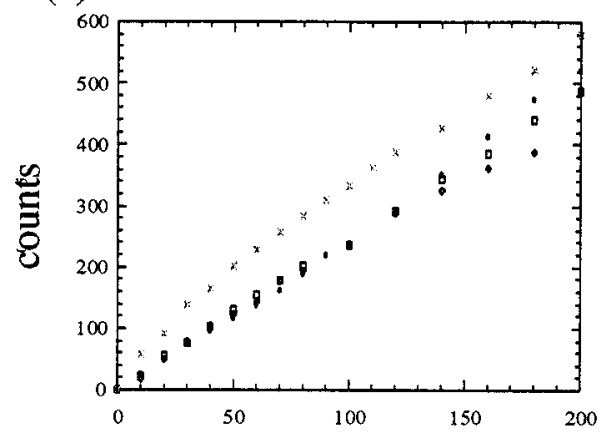

Time (s) (b)

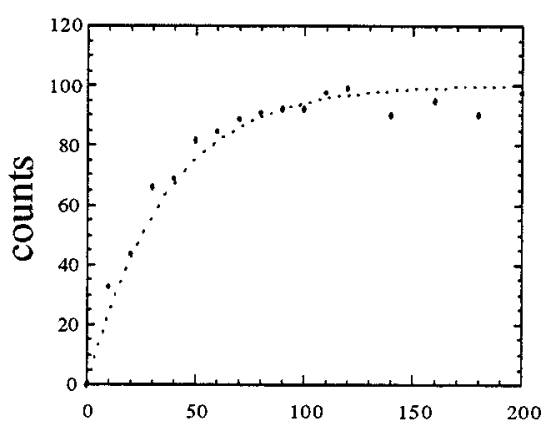

Time (s)
FIG. 6. Measured counts in the Ag activation counter for four shots taken within 100 ps of the peak compression point at $1.7 \mathrm{~ns}$ showing (a) the raw data, (b) the background subtracted result fitted with a $25 \mathrm{~s}$ half life decay curve (dotted line). 
burn-up fraction multiplied by the total energy available from the fuel. The burn up fraction is given by the expression

$$
f_{b} \approx 5.6 \times 10^{3}(\rho R) \exp \left(-19.9 / k T_{\mathrm{keV}}^{1 / 3}\right) / k T_{\mathrm{keV}}^{1 / 3} .
$$

When CD material is substituted for DT and the $\rho R$ of 10 $\mathrm{mg} \mathrm{cm}{ }^{-2}$ from the simulations is used, then the plasma temperature of the CD layer needed to generate $2 \times 10^{4}$ neutrons is $\sim 500 \mathrm{eV}$. This means that $\sim 7 \%$ of the short pulse laser energy was coupled to the plasma to produce this temperature. While this coupling efficiency appears somewhat low, it may be increased by a more suitable match of the range of the fast electrons, which depends upon $I \lambda^{2}$, to the achieved $\rho R$. For instance, at $10^{18} \mathrm{~W} \mathrm{~cm}^{-2}$, the electron temperature is $200-300 \mathrm{keV}$ with a range in plastic of $\sim 140 \mathrm{mg} \mathrm{cm}^{-2}$, somewhat higher than could be achieved in the experiment. An optimization of the coupling of short pulse energy to the compressed plasma will be the subject of further investigation.

\section{SUMMARY}

We have shown that the advanced fast ignitor concepts have effectively traded a plasma physics problem (the holeboring problem) for a hydrodynamics problem (an implosion in the presence of a cone). We have provided the first demonstration, using an open geometry and x-ray backlighting, that the guided foil remains stable during the compression, and that the shape of the guided foil is reproduced in twodimensional hydrodynamic simulations. An integrated conical compression experiment has confirmed that this approach to fast ignition demands detailed investigation.

\section{ACKNOWLEDGMENTS}

The authors gratefully acknowledge the assistance the staff of the Central Laser Facility, together with Eugene Clark and Nigel Rhodes who assisted with the calibration of the neutron activation detector. Special thanks go to the target fabrication groups at the Rutherford Appleton Laboratory (RAL) and Osaka University, and to the micromachining laboratory at RAL for the precise manufacture of the conical targets.

The work was supported by the United Kingdom Engineering and Physical Sciences Research Council, by the Japan Society for the Promotion of Science, and by the British Council.
${ }^{1}$ J. Lindl, Phys. Plasmas 2, 3933 (1995).

${ }^{2}$ M. Tabak, J. E. Hammer, M. E. Glinsky, W. L. Kruer, S. C. Wilks, J. C. Woodworth, E. M. Campbell, and R. J. Mason, Phys. Plasmas 1, 1626 (1994).

${ }^{3}$ S. Atzeni, Jpn. J. Appl. Phys., Part 1 34, 1980 (1995).

${ }^{4}$ S. Atzeni, Phys. Plasmas 6, 3316 (1999).

${ }^{5}$ A. R. Piriz and M. M. Sanchez, Phys. Plasmas 5, 2721 (1998)

${ }^{6}$ R. Kodama, K. Takahashi, K. A. Tanaka, Y. Kato, K. Murai, F. Weber, T. W. Barbee, and L. B. DaSilva, Rev. Sci. Instrum. 70, 543 (1999).

${ }^{7}$ A. J. Mackinnon, M. Borghesi, R. Gaillard, G. Malka, O. Willi, A. A. Offenberger, A. Pukhov, J. Meyer-ter-Vehn, B. Canaud, J. L. Miquel, and N. Blanchot, Phys. Plasmas 6, 2185 (1999).

${ }^{8}$ M. H. Key (private communication).

${ }^{9} \mathrm{~S}$. J. Rose (private communication).

${ }^{10}$ C. N. Danson, J. Collier, L. J. Barzanti, A. Damerell, C. B. Edwards, M. H. R. Hutchinson, M. H. Key, D. Neely, P. A. Norreys, D. A. Pepler, I. N. Ross, P. F. Taday, W. T. Toner, M. Trentleman, F. N. Walsh, T. B. Winstone, and R. W. W. Wyatt, J. Mod. Opt. 45, 1653 (1998).

${ }^{11}$ M. Tatarakis, P. Lee, J. R. Davies, F. N. Beg, A. R. Bell, P. A. Norreys, M. G. Haines, and A. E. Dangor, Phys. Rev. Lett. 81, 999 (1998).

${ }^{12}$ P. A. Norreys, M. Santala, E. Clark, M. Zepf, I. Watts, F. N. Beg, K. Krushelnick, M. Tatarakis, A. E. Dangor, X. Fang, P. Graham, T. McCanny, R. P. Singhal, K. W. D. Ledingham, A. Cresswell, D. C. W. Sanderson, J. Magill, A. Machacek, J. S. Wark, R. Allott, B. Kennedy, and D. Neely, Phys. Plasmas 6, 2150 (1999).

${ }^{13}$ M. Borghesi, A. J. MacKinnon, A. R. Bell, G. Malka, C. Vickers, O. Willi, J. R. Davies, A. Pukhov, and J. Meyer-ter-Vehn, Phys. Rev. Lett. 83, 4309 (1999).

${ }^{14}$ L. Gremillet, F. Amiranoff, S. D. Baton, J.-C. Gauthier, M. Koenig, E. Martinolli, F. Pisani, G. Bonnaud, C. Lebourg, C. Rousseaux, C. Toupin, A. Antonicci, D. Batani, A. Bernardinello, T. Hall, D. Scott, P. Norreys, H. Bandulet, and H. Pepin, Phys. Rev. Lett. 83, 5015 (1999).

${ }^{15}$ M. I. K. Santala, M. Zepf, E. Clark, I. Watts, F. N. Beg, M. Tatarakis, K. Krushelnick, A. E. Dangor, T. McCanny, I. Spencer, R. P. Singhal, K. W. D. Ledingham, S. C. Wilks, A. Machacek, J. S. Wark, R. Allott, R. J. Clarke, and P. A. Norreys, Phys. Rev. Lett. 84, 1459 (2000).

${ }^{16}$ M. H. Key, M. D. Cable, T. E. Cowan, K. G. Estabrook, B. A. Hammel, S. P. Hatchett, E. A. Henry, D. E. Hinkel, J. D. Kilkenny, J. A. Koch, W. L. Kruer, A. B. Langdon, B. Lasinski, R. W. Lee, B. J. MacGowan, A. J. MacKinnon, J. D. Moody, M. J. Moran, A. A. Offenberger, D. M. Pennington, M. D. Perry, T. J. Phillips, T. C. Sangster, M. S. Singh, M. A. Stoyer, M. Tabak, G. L. Tietbohl, M. Tsukamoto, K. Wharton, and S. C. Wilks, Phys. Plasmas 5, 1966 (1998).

${ }^{17}$ G. J. Pert, J. Comput. Phys. 43, 111 (1981).

${ }^{18}$ R. J. Mason, R. J. Fries, and E. H. Farnum, Appl. Phys. Lett. 34, 14 (1979).

${ }^{19}$ S. I. Anisimov, V. I. Vovchenko, A. S. Goncharov, M. F. Ivanov, Yu. S. Kas'yanov, O. V. Kozlov, I. K. Krasyuk, A. A. Malyutin, P. P. Pashinin, A. M. Prokhorov, and L. N. Shchur, Sov. Tech. Phys. Lett. 4, 157 (1978).

${ }^{20}$ S. I. Anisimov, V. E. Bespalov, V. I. Vovchenko, A. N. Dremin, F. I. Dubovitskii, A. P. Zharkov, M. F. Ivanov, I. K. Krasyuk, A. Pashinin, A. M. Prokhorov, V. Ya. Ternovoi, V. E. Fortov, and L. N. Shchur, JETP Lett. 31, 61 (1980).

${ }^{21}$ A. A. Charakhch'yan, J. Appl. Mech. Tech. Phys. 4, 506 (1994).

${ }^{22}$ R. W. Lee and J. T. Larsen, J. Quant. Spectrosc. Radiat. Transf. 56, 535 (1996). 